\section{The relation of schneiderian membrane thickness and membrane perforation in sinus lift procedure: a systematic review}

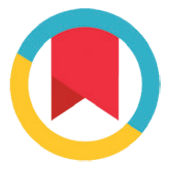

CrossMark

\author{
Sri Oktawati, ${ }^{*}$ Ingrid Neormansyah, Supiaty
}

\title{
Abstract
}

Objective: To evaluate the recent scientific evidence about the relation of schneiderian membrane thickness and sinus lift complications. Methods: A systematic search at pubMed and wiley online library was conducted to identify articles published between January 2013 to December 2017 about schneiderian membrane thickness relation with sinus lift complications. Manual selection of the full text articles was performed and from 133 articles found in initial searches, there were only 3 articles that compatible with the inclusion criteria.
Results: The initial search resulted in 133 articles. All the full text articles were reviewed and selected based on inclusion criteria. There were 3 studies that compatible with inclusion criteria with 395 patients as subjects and 466 sinus lift or sinus augmentation procedures performed.

Conclusion: The schneiderian membrane thickness might affects complications in sinus lift or sinus augmentation procedures, especially perforation of schneiderian membrane.
Department of Periodontic, Faculty of Dentistry, Hasanuddin University, Makassar, Indonesia
"Corresponding to: Sri Oktawati, Department of Periodontic, Faculty of Dentistry, Hasanuddin University, Makassar, Indonesia periounhas_sri@yahoo.com

Received: 17 May 2018

Revised: 17 May 2018 Accepted: 9 July 2018 Available Online 1 August 2018

Keywords: Complications, Maxillary sinus floor augmentation, Schneiderian membrane, Sinus augmentation therapy

Cite this Article: Oktawati S, Neormansyah I, Supiaty. 2018. The relation of schneiderian membrane thickness and membrane perforation in sinus lift procedure: a systematic review. Journal of Dentomaxillofacial Science 3(2): 67-69. D0I: 10.15562/jdmfs.v3i2.737

\section{Introduction}

Implants treatment in atrophic posterior maxilla is one of the most challenging cases for an implantologist. ${ }^{1}$ Dr. O. Hilt Tatum (US) pioneered in developing the first sinus lift operation in 1974 and sinus graft operation in 1975. Recently, sinus augmentation has been widely performed to augment vertical deficient ridge. ${ }^{2}$ The bone height deficiency in maxillary posterior caused by sinus maxillary expansion or atrophy process. ${ }^{3}$ Sinus maxillary membrane, known as schneiderian membrane is the membrane that lines on the inner part of the maxillary sinus cavities. ${ }^{4}$ Schneiderian membrane was $1.32+/-0.87 \mathrm{~mm}$. Perforation rate was lowest $7.14 \%$ Schneiderian Membrane Thickness (SMT) has been considered as a key factor that influence sinus lift complications ${ }^{5}$ Nonetheless, sinus augmentation has been proven to be a safe procedure with high success rate. ${ }^{6,7}$

A major complication in sinus lift and augmentation procedure is schneiderian membrane perforation. Schneiderian membrane's elastic feature and quality affect the possibility of perforation. This complication increases the possibility of side effect for graft loss, an infection that will cause an interference in sinus function, implant survival and the clinical outcome of sinus augmentation ${ }^{5}$ Several studies about sinus lift concluded that there is a relation between SMT and sinus lift complications. ${ }^{4,6}$ The aims of this systematic review is to evaluate the recent scientific evidence about the SMT relation with sinus lift possibility of complications.

\section{Methods}

This article was written and conducted according to Preferred Reporting Items for Systematic Reviews and Meta Analysis (PRISMA) guidelines to report studies that compare SMT with sinus lift complications. ${ }^{8}$ The Population Interventio Comparison Outcome (PICO) questions for this article were the patients who are going to undergo an implant treatment on posterior maxilla and sinus lift procedure. ${ }^{9}$

A systematic search was made to identify articles published between January 2013 and December 2017 with the keywords used were "Schneiderian membrane thickness" and "sinus lift complications". The article search was limited to English articles and full-text availability. The title and fulltext were then reviewed to find the relevant studies. After searching with those keywords, there were 133 initial articles found but only 3 studies met the inclusion criteria.

The inclusion criteria used for this article were full text articles in English that were published between January 2013 and December 2017 and the studies subject were human. Those articles were classifying schneiderian membrane thickness and also including informations about complication of 
sinus lift. While the exclusion criteria used for this systematic review were any articles that discuss about systematic review and meta analysis and the studies were in animal or in vitro or cadaver.

The authors (SO, IN and S) were using specific keywords to review and select the articles independently by screening the title. The full texts of the article that compatible with the topic of this systematic review was obtained and analyzed with the inclusion criteria that has been determined to set the final list of articles. The reviewers (SO, IN and $\mathrm{S}$ ) independently extracted the data that is compatible with the inclusion criteria. After that, the final articles were discussed until there were no disagreement. The data that were extracted from the selected articles were author(s), year of publication, number of patients, age range (or mean range), SMT classification and complications.

\section{Results}

Figure 1 the initial search resulted in 133 articles. After screening the title and reviewing the full text articles, 128 articles were excluded with the

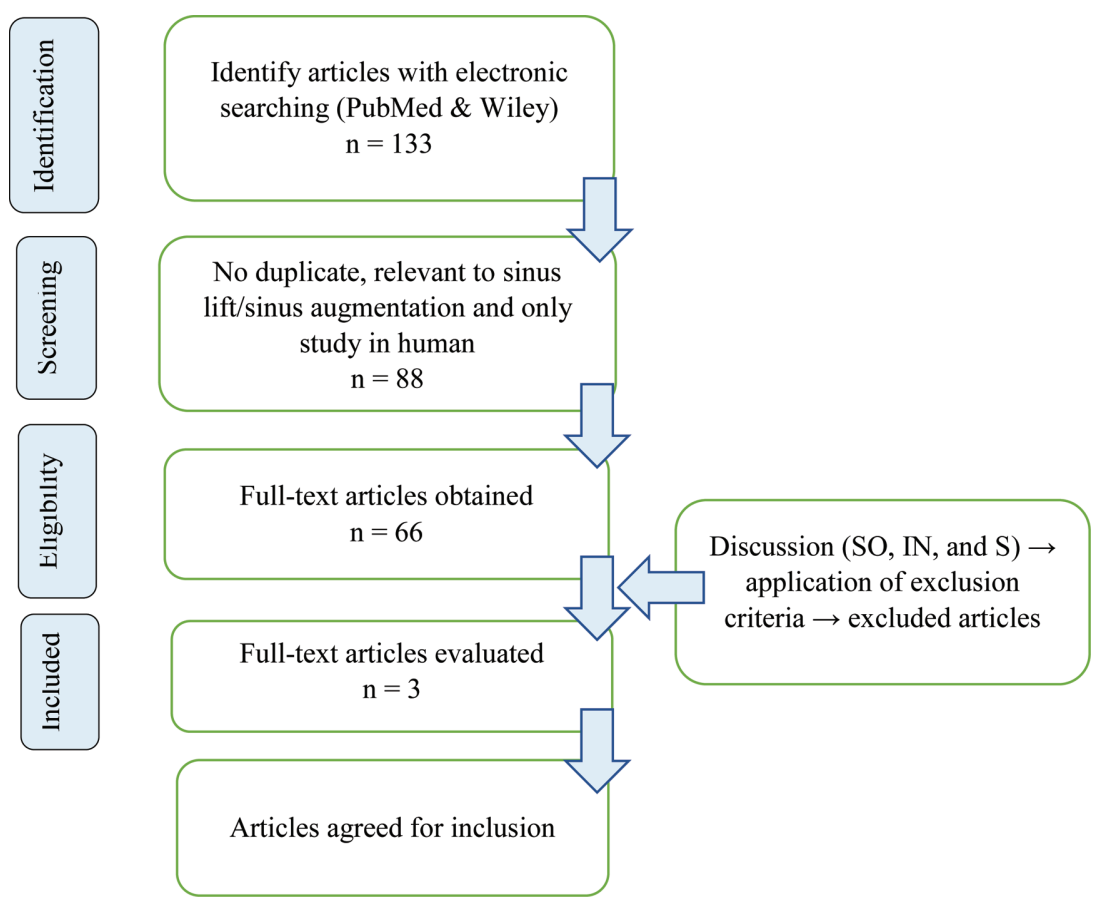

following specification: 2 articles were duplicate, 13 articles were not relevant to sinus lift or sinus augmentation procedure, 13 articles were literature review or systematic review and meta analysis, 12 full text articles cannot be obtained, 17 articles studied about animal or cadaver or models, 10 articles were part of textbook, 61 articles did not study about SMT or complications or both and 2 articles did not classify SMT. After screening, reviewing and selecting those articles, only 3 articles met the inclusion criteria.

Table 1 showed that all of the studies were a retrospective type. The studies were done in 2014, 2015 and 2016. The patient's age was range between 20-79 years. Total patients that undergo sinus lift were 395 , with 466 sinus lift cases. The schneiderian membrane thickness was range between 0 to more than $11.8 \mathrm{~mm}$. The most perforated membrane compared with all class in these studies is the schneiderian membrane with > $2 \mathrm{~mm}$ thickness which has 6 perforated membranes in 22 cases.

\section{Discussion}

All the studies included were conducted in the past 5 years and were a retrospective study. A total of 395 patients were included in these studies with 466 sinus lift cases. These studies only reported about perforation of schneiderian membrane as sinus lift complication and no other else.

Wen et al. ${ }^{6}$ and Lin et al. ${ }^{4}$ measured and divided the schneiderian membrane thickness into 3 groups less than $1 \mathrm{~mm}, 1 \mathrm{~mm}$ to less than $2 \mathrm{~mm}$ and more than $2 \mathrm{~mm}$ with mean thickness $1.78 \pm 1.99$ $\mathrm{mm}$ in first study and $1.32 \pm 0.87 \mathrm{~mm}$ in the second study. It was using crestal approach technique and using lateral window technique. The complication of sinus lift reported in both studies is perforated of Schneiderian membrane, with the most perforated one is in the more than $2 \mathrm{~mm}$ group in study about $20.83 \%$ and $27.27 \%$. The studies also reported that the less perforated group is the 1 to less than $2 \mathrm{~mm}$ about $13.85 \%$ study.

The methods and results in Rajani et al. ${ }^{10}$ study divided the schneiderian membrane into 4 groups:

Figure 1 PRISMA flow chart

Table 1 Description data from the 3 included studies that reported on schneiderian membrane perforation

\begin{tabular}{|c|c|c|c|c|c|c|c|}
\hline & Age (years) & $\begin{array}{l}\text { Patients/ } \\
\text { Sinus Lift } \\
\text { (n) }\end{array}$ & $\begin{array}{c}\text { SMT }<1 \mathrm{~mm} \\
\text { perforation } \\
(\mathrm{n} \%)\end{array}$ & $\begin{array}{c}\text { SMT } 1-<2 \mathrm{~mm} \\
\text { perforation } \\
(\mathrm{n} \%)\end{array}$ & $\begin{array}{c}\text { SMT > } 2 \text { mm } \\
\text { perforation } \\
(n \%)\end{array}$ & $\begin{array}{c}\text { SMT > } 4 \mathrm{~mm} \\
\text { perforation } \\
\text { (n\%) }\end{array}$ & $\begin{array}{c}\text { Number of } \\
\text { perforation } \\
\text { (n\%) }\end{array}$ \\
\hline Wen et al. & $40-64$ & $122 / 185$ & $13 / 72(18)$ & 9/65 (13.8) & $10 / 48(20.8)$ & $\mathrm{N} / \mathrm{A}^{*}$ & $22 / 185(11.9)$ \\
\hline Lin et al. & $29-78$ & $73 / 81$ & $7 / 34(20.6)$ & $1 / 25(4)$ & $6 / 22(27)$ & $\mathrm{N} / \mathrm{A}^{*}$ & 14/81(17.3) \\
\hline Rapani et al. & $20-79$ & $200 / 200$ & $12 / 52(23.1)$ & $4 / 35(11.4)$ & $0 / 67(0)$ & $0 / 46(0)$ & $16 / 200(8)$ \\
\hline
\end{tabular}

${ }^{*}$ Not Available 
$0 \mathrm{~mm}$ or too thin to record (type I), less than $2 \mathrm{~mm}$ (type II), 3 and $4 \mathrm{~mm}$ (type III), and more than $4 \mathrm{~mm}$ (type IV). The subject of this study also treated with 2 different techniques; lateral window technique in 100 patients and crestal approach technique in 100 patients. Type I group was the most perforated group with 5 perforations in lateral window technique and 7 perforations in crestal approach technique. The type I group total perforation for both techniques is 12 out of 52 sinus lift which is $23.08 \%$, while type III and type IV had no perforation for both techniques. ${ }^{10}$

Some studies reported that there were certain schneiderian membrane thickness that shown to be vulnerable to intra operative membrane perforation, but thick membrane is not preventing membrane perforation as the resistance under elastic forces is not better than the thinner one. ${ }^{11,12}$ Complex sinus wall morphology, the presence of sinus septa and force applied to the membrane might also increase the chance of membrane perforation. The prevalence and morphology of sinus septa were first described in $1910 .{ }^{13}$ Schneiderian membrane perforation might occur at force more than $7.3 \mathrm{~N} /$ $\mathrm{cm}^{12}$

\section{Conclusion}

From the results of those studies, conclusions can be made within the limitation of this review is the schneiderian membrane thickness might affect complications in sinus lift or sinus augmentation procedures, especially perforation of schneiderian membrane. This study found a positive relation between schneiderian membrane thickness with perforation of schneiderian membrane. There are many limitations in this article, therefore more research with larger sample size and better methodology research are necessary to conclude a better an accurate results.

\section{Acknowledgment}

I would like to thank all the Lecturer Department of Periodontology, M. Ruslin, Acing and Adytha for guiding and helping me until the completion of this article.

\section{Conflict of Interest}

The authors report no conflict of interest.

\section{References}

1. Al-Dajani M. Incidence, risk factors and complications of schneiderian membrane perforation in sinus lift surgery: A meta-analysis. Implant Dent 2016;25: 409-415.

2. Insua A, Monje A, Chan HL, et al. Accuracy of Schneiderian membrane thickness: a cone-beam computed tomography analysis with histological validation. Clin Oral Implants Res 2017;28: 654-661.

3. Ruslin M. Lateral window and transalveolar osteotomy sinus lifting technique. J Dentomaxillofac Sci 2011;10: 111-115.

4. Lin YH, Yang YC, Wen SC, et al. The influence of sinus membrane thickness upon membrane perforation during lateral window sinus augmentation. Clin Oral Implants Res 2016;27: 612-617.

5. Monje A, Diaz KT, Aranda L, et al. Schneiderian membrane thickness and clinical implications for sinus augmentation: a systematic review and meta-regression analyses. J Periodontol 2016;87: 888-899.

6. Wen SC, Lin YH, Yang YC, et al. The influence of sinus membrane thickness upon membrane perforation during transcrestal sinus lift procedure. Clin Oral Implants Res 2015;26: 1158-1164.

7. Vanderburg NJ, Kolhatkar S, Bhola M. Management of a large schneiderian membrane perforation and repair of the missing maxillary lateral wall using a collagen barrier alone in a patient with human immunodeficiency virus/acquired immunodeficiency syndrome. Clin Adv Periodontics 2015;5: 116-123.

8. Liberati A, Altman DG, Tetzlaff J, et al. The PRISMA statement for reporting systematic reviews and metaanalyses of studies that evaluate healthcare interventions: explanation and elaboration. BMJ 2009;339.

9. Long M, Tao S, Vega D, et al. Extracting PICO sentences from clinical trial reports using supervised distant supervision. JMLR 2016;8: 444-454.

10. Rapani M, Rapani C, Ricci L. Schneider membrane thickness classification evaluated by cone-beam computed tomography and its importance in the predictability of perforation. Retrospective analysis of 200 patients. Br J Oral Maxillofac Surg 2016;54: 1106-1110.

11. Insua A, Monje A, Chan HL, et al. Association of inflammatory status and maxillary sinus schneiderian membrane thickness. Clin Oral Investig 2017: 1-10.

12. Insua A, Monje-Gil F, García-Caballero L, et al. Mechanical characteristics of the maxillary sinus Schneiderian membrane ex vivo. Clin Oral Investig 2017: 1-7.

13. Schwarz L, Schiebel V, Hof M, et al. Risk factors of membrane perforation and postoperative complications in sinus floor elevation surgery: review of 407 augmentation procedures. J Oral Maxillofac Surg 2015;73: 1275-1282.

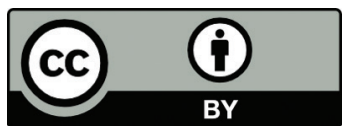

This work is licensed under a Creative Commons Attribution 